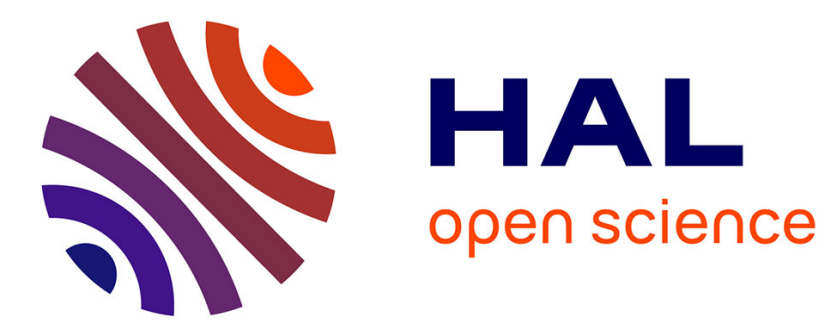

\title{
Les traits définitoires de l'humain dans la série télévisée Doctor Who
}

\author{
François-Ronan Dubois
}

\section{To cite this version:}

François-Ronan Dubois. Les traits définitoires de l'humain dans la série télévisée Doctor Who . Élaine Després; Hélène Machinal. Posthumain : frontières, évolutions, hybridités, Presses Universitaires de Rennes, pp.181-191, 2014, 978-2-7535-3374-5. halshs-01346159

\section{HAL Id: halshs-01346159 \\ https://shs.hal.science/halshs-01346159}

Submitted on 22 Jul 2016

HAL is a multi-disciplinary open access archive for the deposit and dissemination of scientific research documents, whether they are published or not. The documents may come from teaching and research institutions in France or abroad, or from public or private research centers.
L'archive ouverte pluridisciplinaire HAL, est destinée au dépôt et à la diffusion de documents scientifiques de niveau recherche, publiés ou non, émanant des établissements d'enseignement et de recherche français ou étrangers, des laboratoires publics ou privés.

\section{(1) $\$(0)$}

Distributed under a Creative Commons Attribution - NonCommercial - ShareAlikel 4.0 
"Les traits définitoires de l'humain dans la série télévisée Doctor Who ». Posthumain : frontières, évolutions, hybridités. Élaine Després et Hélène Machinal, dir. Rennes : Presses Universitaires de Rennes, 2014. 181-191.

\section{Les traits définitoires de l'humanité dans la série télévisée Doctor Who (2005)}

François-Ronan Dubois

À l'heure où les modifications biotechnologiques prennent part à l'imaginaire collectif $\mathrm{du}$ corps et où les cyborgs, les membres mécaniques et les intelligences artificielles s'échappent du pré carré de la science-fiction littéraire et cinématographique pour atteindre des produits médiatiques plus largement diffusés, à l'heure, donc, où la post-humanité cesse d'être uniquement l'inquiétante prophétie ou la prédiction radieuse d'un petit groupe de spécialistes pour prendre part à l'actualité et ses représentations communes, les récits sciencefictifs acquièrent une valeur littérale et prospective. Littérale, parce que ne s'y lit plus uniquement la métaphore d'un présent ; prospective, parce qu'ils développent, dans un univers de rationalité restreinte, les possibilités du monde actuel. La science-fiction cesse ainsi d'habiter aussi naturellement qu'elle paraissait le faire le territoire de l'imaginaire pour devenir référentielle. C'est à ce titre, en qualité de récit prospectif sérieux susceptible de décrire avec une certaine exactitude un avenir prochain, que la science-fiction est convoquée dans le débat politique. ${ }^{1}$

Cependant, cette fonction prospective et virtuellement référentielle n'épuise pas le discours science-fictif. Elle ne saurait par exemple rendre compte de manière satisfaisante des récits, qu'ils soient audiovisuels ou scripturaux, qui n'accordent que peu de place au traitement des mécanismes internes des sciences ou dont l'univers fictif est organisé par des sciences imperméables à la rationalité habituelle. En d'autres termes, lorsque le récit cesse de faire référence à la science telle que nous la concevons, dans ses procédures si ce n'est dans ses résultats et ses objets, l'examen de son éventuelle puissance prospective risque fort d'être la source d'une vive déception. ${ }^{2}$ Je fais ici l'hypothèse que, dans ces récits, l'anticipation scientifique superficielle est un moyen de définition du présent par les marges; en d'autres termes, pour le cas qui nous occupe dans ce volume, que le cyborg n'y constitue pas une réflexion sur la post-humanité, mais une tentative de définition (et donc de naturalisation) de l'humanité.

1 CATEllin, S., " Le recours à la science-fiction dans le débat public sur les

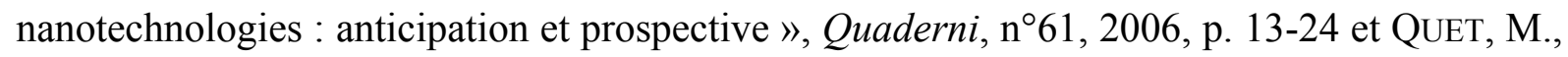
" La critique des technologies émergeantes face à la communication promettante. Contestations autour des nanotechnologies », Réseaux, n¹73-174, 2012-2013, p. 271-301. Voir aussi la contribution de Mathieu Quet dans ce volume et l'ensemble des travaux de Sylvie Allouche.

${ }^{2}$ Comme le rappelait Sylvie Allouche, à Arras en novembre 2012 à l'occasion du colloque Poétiques du merveilleux : fantastique, science-fiction, fantasy (littérature et arts visuels), sous la direction d'Anne Besson et d'Evelyne Jacquelin, cette inefficacité prospective peut motiver un remaniement des classifications génériques et l'exclusion du champ de la sciencefiction de ce type de récits. 
C'est dans cette perspective que je propose d'aborder ici l'un de ces récits audiovisuels : la série télévisée Doctor Who, telle qu'elle est diffusée dans sa version moderne sur les écrans britanniques depuis 2005. Originellement créée en 1963, interrompue en 1989, la série a repris sans rupture narrative au vingt-et-unième siècle sous la direction de Russell $\mathrm{T}$. Davies puis de Steven Moffat. Elle met en scène les aventures du Docteur, extraterrestre anthropomorphe millénaire, et de ses compagnes de voyage humaines, qui traversent le temps et l'espace dans le TARDIS pour combattre les extraterrestres. La série connaît une popularité unique dans le paysage audiovisuel britannique, à la fois en termes quantitatifs et qualitatifs. Elle fait l'objet, depuis les années 1980, d'une attention continue de la part d'universitaires issus de disciplines variées. ${ }^{3}$

La technologie à l'œuvre dans ces nouveaux Doctor Who est en effet parfaitement fantaisiste et elle ressort bien plutôt de la rationalité ésotérique de la magie que de la rationalité exotérique de la science. ${ }^{4}$ Les explications du Docteur, souvent nébuleuses, n'éclaircissent nullement le fonctionnement interne des techniques et des procédures représentées et c'est plus généralement la techno-science en tant qu'objet imaginaire que les disciplines scientifiques en tant que processus de constitution du savoir qui fait le thème de la série. Cette disposition a une double conséquence. D'une part, l'enjeu des histoires technoscientifiques proposées par le récit n'est pas d'ordre épistémologique mais éthique et moral. D'autre part, le récit est moins prospectif que réflexif. C'est à partir de ces deux caractéristiques que j'examinerai ici le traitement thématique et visuel de l'humanité dans la série, via les marges de la post-humanité.

J'étudierai dans un premier temps quelques cas de post-humains et je tenterai d'isoler les traits définitoires de l'humanité que leur traitement propose. Il s'agira ensuite de replacer ces traits dans un système axiologique plus vaste, pour comprendre l'évaluation qui en est faite. A partir de cette construction, j'essayerai de déterminer une éthique du savoir à l'œuvre dans la série qui ne soit pas nécessairement négative.

\section{Les post-humains ${ }^{5}$}

Par post-humain, j'entends ici un être humain dont l'existence corporelle est affectée par un ou plusieurs processus techno-scientifiques. Cette définition exige, pour les cas présents dans le corpus considéré, une subdivision fondamentale, qui fasse la distinction entre les humains modifiés par la techno-science et les humains créés par la techno-science. Les premiers sont évidemment représentés à deux stades de leur existence corporelle, c'est-à-dire que le récit audiovisuel propose deux images différentes de leur être : leur apparence humaine puis leur apparence post-humaine. Les seconds sont essentiellement post-humains : il n'existe

3 Voir notamment l'ouvrage fondateur : Tulloch, J. et Alvarado, M, Doctor Who : The Unfolding Text, Londres, Macmillian Publishers, 1983.

${ }^{4}$ Voir l'analyse comparée des différentes périodes de l'histoire de la série dans : ORTHIA, L., «Enlightenment was the choice : Doctor Who and the democratisation of science », Canberra, Thèse de doctorat, 2010.

5 Pour une analyse qui contredise la mienne, on pourra se reporter à : CRANNY-FrANCIS, A., «Mediating Human-technology Relationships : Explorations of Hybridity, Humanity and Embodiment in Doctor Who », Forum, n8, 2009, en ligne. 
pas pour eux d'état antérieur à l'intervention de la techno-science. La typologie peut être encore affinée. ${ }^{6}$

On peut classer dans le premier groupe quelques personnages remarquables : Cassandra O'Brien (1.2 et 2.1), les Cybermen (passim), le professeur Lazarus (3.6), les Patchwork People (6.3), les Toclafanes (3.11-12-13), Oswin-Dalek (7.1) et Gunslinger (7.3). ${ }^{7}$ Il est encore possible de les repartir en trois catégories : Cassandra et Lazarus ont volontairement subi leurs transformations ; Oswin, Gunslinger et les Cybermen (à quelques exceptions près) sont des victimes malheureuses, quant aux Patchwork People et aux Toclafanes, leur degré d'implication dans les processus qui les ont modifiés est incertain. On peut également répartir le groupe en deux catégories, selon la discipline employée. Cassandra, Lazarus et les Patchwork People ont subi des interventions biologiques et génétiques ; les autres cas relèvent de la cybernétique. Le second groupe est également varié. On y retrouve les cobayes de New Earth (2.1), le Dalek-Humain (3.4-5), Jenny (4.6), Bracewell (1.3) et les Gangers (6.5-6). On observe ici une nette prédominance de la biotechnologie, qui concerne tous les individus du groupe, exception faite du robot Bracewell. Puisqu'ils sont entièrement créés par la technologie, tous les cas sont involontaires.

En d'autres termes, il n'existe pas une très grande variabilité entre les différents cas : les processus impliqués sont peu nombreux et les situations narratives assez semblables. La post-humanité n'est pas l'objet d'une exploration systématique ni même d'une déclinaison partielle. Tous les cas valent comme contre-exemple de l'humanité dont ils ont été exclus ou à laquelle ils se sont volontairement soustraits. En contrepoint, l'humanité, des périodes reculées de Pompéi aux survivants de la fin de l'univers, se présente comme un ensemble stable, homogène, qui ne subit pas d'évolution spontanée : le citoyen latin de «The Fires of Pompei » (4.3) ressemble en tout point au militaire du cinquante-et-unième siècle. La nature humaine est donc un substrat stable dont la post-humanité ne constitue pas l'évolution inévitable : elle se présente comme une perversion du paradigme.

Les critères subjectifs et psychologiques deviennent alors plus pertinents, pour le classement des individus post-humains, que les critères objectifs et techniques. En d'autres termes, ce qui distingue ces individus les uns des autres, ce ne sont pas tant les processus techno-scientifiques dans lesquels ils sont impliqués que les restes psychologiques d'humanité dont ils sont susceptibles de faire preuve. La condition post-humaine devient alors le test des critères fondamentaux de l'appartenance à l'humanité. Pour mieux comprendre, on peut observer quelques cas limites.

Les Cybermen sont des robots dans lesquels est implanté le cerveau d'un être humain. Dotés d'un corps a priori entièrement métallique, ils sont produits par des machines de torture qui extraient le cerveau d'un humain encore vivant et le fixent sur la machine. Ce corps mécanique comporte un inhibiteur d'émotions, qui transforme chaque Cyberman en un parfait automate, dénué de personnalité propre. Pour la plupart d'entre eux, la limite entre le cyborg et le robot est franchie. Il arrive cependant qu'un être humain puisse conserver sa personnalité au cours du processus de transformation et même après son accomplissement : c'est le cas

${ }^{6}$ Je ne propose pas ici un relevé exhaustif de tous les personnages post-humains, dont certains ne font une apparition que ponctuelle et ne jouent qu'un rôle restreint dans l'intrigue.

${ }^{7}$ Le premier numéro indique la saison, le second l'épisode. Pour les titres, les résumés et les fiches techniques, on pourra se reporter à la section consacrée à Doctor Who du site Internet Movie Database : < http://www.imdb.fr/title/tt0436992/>. 
dans « Army of Ghosts - Doomsday » (2.12-13) et dans "Closing Time » (6.12). Dans la première histoire, une fervente patriote continue à défendre son pays après avoir été changée en Cyberwoman ; dans l'autre, l'amour d'un père pour son fils interrompt le processus de transformation. ${ }^{8}$ De la même manière, l'épisode " Victory of the Daleks " (1.3) met en scène le savant Bracewell qui, pendant la Seconde Guerre Mondiale, aide Churchill à repousser les attaques allemandes. Mais le Docteur découvre que Bracewell est un robot construit par des extraterrestres maléfiques. Le robot contient une bombe susceptible de détruire la planète. Fort heureusement, la compagne du Docteur, Amelia Pond, réussit à convaincre Bracewell de son humanité en lui montrant qu'il est capable de ressentir l'amour.

Les sentiments sont donc le premier critère de séparation entre l'humain et la machine. Dans l'univers science-fictif, la faculté de raisonner cesse d'être le propre de l'être humain et la raison est au contraire l'apanage de l'esprit mécanique. Or, cet esprit est incapable d'intégrer la complexité du monde, qui ne peut être l'objet que d'une approche sensible et émotive. Si l'être humain est supérieur à la machine, si, au sein du récit, il l'emporte sur les robots et les cyborgs, c'est parce qu'il est irrationnel et, en quelque manière, inférieur. Cette évolution positive de l'émotion au détriment de la raison est le premier signe d'un éloge général de l'infirmité, à l'œuvre dans d'autres cas.

Les situations inverses conduisent en effet aux mêmes conclusions. Si un humain transformé involontairement en post-humain peut conserver son humanité grâce à sa propre infirmité, c'est, selon une même logique, la volonté de dépasser l'infirmité de leur condition qui finit par exclure de l'humanité ceux qui se soumettent volontairement à de semblables processus. Ainsi, lorsque l'inventeur des Cybermen est absorbé par sa propre création (« The Age of Steel », 2.6), il perd toute personnalité et précisément ce qui le distinguait d'entre tous : son génie. Quand le professeur Lazarus souhaite conquérir la vie éternelle en modifiant son code génétique, il se transforme en créature monstrueuse et Cassandra O'Brien, qui poursuit un semblable rêve, est réduite à un simple lambeau de peau. C'est le désir d'immortalité qui guide ces trois personnages. L'être humain ne doit donc pas tenter de dépasser sa propre condition et il n'existe aucune place, dans les nouveaux Doctor Who, pour le rêve transhumaniste d'une existence augmentée ${ }^{9}$; tout au contraire, de pareilles tentatives servent de repoussoirs et ne se soldent jamais que par l'aliénation de celui qui s'y prête, offrant ainsi un récit dont la morale est dépourvue de toute ambiguïté : il faut se satisfaire de son existence limitée.

Si la morale est sans ambiguïté, c'est que la construction discursive de l'idéologie à travers les dialogues et l'organisation narrative est encore soutenue par la représentation iconographique des individus en question. Pour chacun des cas qui oscillent entre humanité et inhumanité, la série propose deux images concurrentes : celle du corps habituel du comédien et celle de ce même corps modifié par les accessoires prosthétiques et les techniques de postproduction. Ainsi en va-t-il des Gangers, corps biologiques artificiels qui servent de doubles à des techniciens opérant dans des milieux dangereux. Lorsque les Gangers développent une personnalité propre, des émotions, une sensibilité, le comédien incarne simplement le double

${ }^{8}$ Pour une analyse de la représentation des Cybermen dans la première série, se reporter à : Geraghty, L., "From Balaclavas to Jumpsuits : The Multiple Histories and Identities of Doctor Who's Cybermen », Atlantis, vol. 30, n¹, 2008, p. 85-100.

${ }^{9}$ Sur cette question, voir par exemple dans ce volume la contribution de Pierre CassouNoguès. 
de son premier personnage, qui devient ainsi parfaitement interchangeable avec l'être humain. En revanche, lorsque certains Gangers, habités par une déraisonnable soif de puissance, repoussent les limites de l'humanité, leurs corps se transforment et l'image est retravaillée par les effets spéciaux : le monstre est né. Le cas le plus remarquable d'un semblable processus est sans doute celui des cobayes de New Earth, dans l'épisode du même nom. Créés par ingénierie biologique pour servir de cobayes médicaux, les créatures s'échappent de leur cuve d'incubation et errent dans le complexe hospitalier. Le maquillage de pestiféré, la démarche titubante, le jeu d'acteurs limité, tout est fait pour rappeler le zombie. En revanche, quand le Docteur soigne ces malheureux cobayes, leur peau reprend un aspect sain, leur démarche s'assouplit er leur visage exprime pour la première fois des émotions.

En somme, narrativement, discursivement et visuellement, la série met en place un système idéologique au sein duquel la post-humanité est systématiquement condamnée, parce qu'elle outrepasse les limites de la condition humaine. Les traits définitoires de l'humanité sont alors essentiellement négatifs, exprimant non une puissance, mais une infirmité : il s'agit de l'irrationalité, de l'ignorance et de la mort.

\section{Le bien-et-mal}

On le voit, l'opposition entre humains et post-humains n'est, dans Doctor Who, qu'une modalité de l'opposition entre bien et mal. L'humanité est du côté du bien ; ce de quoi les post-humains participent, du côté du mal. Or, cette opposition est incarnée narrativement dans le récit par la confrontation entre deux séries de personnages, dont les uns sont les plus purs représentants du bien (et de l'humanité) et les autres les plus purs représentants du mal (et de l'inhumanité) ; cette opposition, c'est celle des compagnes successives du Docteur et des Daleks.

Comme le suggère le diptyque déjà évoqué "Army of Ghosts Doomsday »(2.12-13), les Daleks constituent l'expression paroxystique d'un mal dont les Cybermen sont un état intermédiaire. Poulpes extraterrestres gélatineux enfermés dans un exosquelette en forme de poivrière géante, avec ventouse et rayon laser, les Daleks sont les créatures les plus dangereuses de l'univers, animées par un seul sentiment : la haine de tout ce qui n'est pas Dalek. Leur but (et leur principale activité) est par conséquent l'extermination de tout autre forme de vie et subséquemment la conquête de toutes les réalités. Les Daleks sont également un sommet de la techno-science : très rationnels, brillants roboticiens, brillants généticiens, ingénieurs remarquables, ils construisent vaisseaux, machines et créatures pour mener à bien leurs sinistres projets. Alors que les Cybermen sont toujours en quelque manière marqués par leur origine humaine, les Daleks ne se laissent jamais déstabiliser. A l'opposée du spectre axiologique, les compagnes du Docteur incarnent l'humanité dans son état le plus parfait. Généralement jeunes et jolies, elles vivent d'abord dans la Grande-Bretagne de l'époque contemporaine et sont choisies par le mystérieux aventurier pour leur sollicitude sans limite, leur bonté d'âme, leur courage, leur enthousiasme poétique à l'idée de découvrir le vaste univers. Rôle principal, dont l'importance tend souvent à supplanter celle du Docteur, elles sont l'objet d'innombrables intrigues et de gros plans, qui permettent aux comédiennes de déployer la gamme complète de leurs émotions, offrant ainsi un contraste remarquable avec l'immobilité métallique des Cybermen et des Daleks d'une part, et avec le jeu codifié du Docteur d'autre part. 
Dans « Asylum of the Daleks » (7.1), le personnage d'Oswin devient l'incarnation de cette dichotomie. Elle est d'abord présentée comme une jeune femme séduisante et brillante, enfermée dans une capsule de sauvetage de son vaisseau spatial, enterrée sous terre, dans une planète infestée de Daleks, contre les assauts desquels elle lutte quotidiennement. L'épisode offre une grande extension aux scènes où l'actrice, dans le huis-clos d'un espace exigu, déploie son charme, ses mimiques et livre les réparties spirituelles et fantasques qui signalent son inventivité humaine. Mais à la fin de l'épisode, le spectateur découvre qu'il ne survit d'Oswin que l'esprit : son corps a été transformé en Dalek. Peu après cette première image de la jeune femme en Dalek, les instincts de sa nouvelle race font surface. Quelques épisodes plus tard, dans « The Snowmen » (7.6), le récit révèle qu'Oswin sera la nouvelle compagne du Docteur, succédant ainsi à Amelia Pond. Le traitement visuel des compagnes, qui offre une large place aux expressions autant qu'aux discours, souligne leur rôle émotif; leur traitement discursif au sein des répliques du Docteur, qui loue constamment leur précieuse sensibilité et fait d'elles les parangons de l'humanité, donne à ce rôle émotif une valeur moralement décisive.

A l'inverse, les Daleks constituent l'incarnation du mal. Leur enfermement dans un exosquelette les rend à peu près interchangeables, de sorte qu'ils apparaissent presque toujours comme les représentants d'une espèce plutôt que comme des individus. Dès leur première apparition dans le récit, dans « Dalek »(1.6), le Docteur les décrit comme ses ennemis les plus dangereux et les plus détestés. Il n'existe aucune place pour la nuance dans leur évaluation par le héros de la série : comme les compagnes, les Daleks sont une fonction morale ou, pour reprendre le terme par lequel Roland Barthes décrivait les catcheurs, un « signe métaphysique ». Dès lors, toutes les caractéristiques qui leur appartiennent en propre sont axiologiquement dévaluées : elles se rapportent au mal. La plupart de ces caractéristiques ne sont pas faites pour surprendre : haine, désir de suprématie, violence, sournoiserie, etc. Mais on y trouve également le désir de survivre à tout prix, une intelligence redoutable et une maîtrise techno-scientifique uniquement dépassée par celle du Docteur. Grâce à cette caractérisation, l'histoire des Daleks devient une raison suffisante pour opposer à n'importe quel développement techno-scientifique : une méfiance de principe. Les Daleks sont l'aboutissement possible du processus post-humain.

Cette organisation est rendue plus explicite encore par la réapparition, au sein de la nouvelle série, dans le diptyque « The Stolen Earth - Journey's End » (4.12-13), de Davros, créateur des Daleks. Davros est un être humanoïde, mutilé, infirme, comme l'était Lubic, le créateur des Cybermen, qui se déplace dans un engin motorisé semblable à un Dalek. C'est à partir des cellules de son propre corps que le savant fou cultive de nouveaux Daleks. Avec le spectacle visuel du corps de Davros palpitant de Daleks en devenir et enfermé dans son appareillage prosthétique, la série lie visuellement l'histoire des Daleks aux autres représentations, à la fois biogénétiques et cybernétiques, de la post-humanité. Ainsi, le système de signification a deux extrémités fortes qui en informent les variations : les compagnes émotives, soutenues par l'art de la comédienne, incarnent les valeurs parfaites de l'humanité, tandis que les Daleks, soutenus par l'art du décorateur et du maquilleur, incarnent les dangers de la post-humanité.

\section{L'éthique du savoir}


Ce dispositif idéologique, on le voit bien, n'est pas sans poser de problèmes. En effet, il entend figer l'humanité dans une essence bien définie et entièrement stable, de sorte que tout progrès paraît exclu de l'horizon de l'espèce. Au sein de la diégèse, cette fixité est encore renforcée par la répartition des schémas narratifs que je viens d'évoquer à divers endroits de la chronologie : les mêmes problèmes et les mêmes résolutions sont présentés dans le passé, dans le présent et dans le futur. Le voyage temporel conduit alors à la négation de la progression possible de l'humanité ; coupée de la justification d'un finalisme scientiste, la post-humanité ne peut plus être alors comprise comme un pas avant : elle est un pas de côté, ce que j'ai appelé en en première partie une perversion du paradigme.

Or, le consensus critique veut, depuis les études fondatrices de Tulloch et Alvarado jusqu'aux récents travaux de Lindy Orthia, que l'idéologie dominante de Doctor Who soit celle d'un humanisme progressiste. Il est vrai que la thèse d'Orthia évoque les rapports parfois difficiles du programme avec la science et qu'Alan McKee soulignait, il y a quelques années, que l'histoire était reçue avec le même enthousiasme par tous les spectateurs, d'un bout à l'autre du spectre politique. ${ }^{10}$ Cette ambiguïté politique n'est d'ailleurs pas propre à Doctor Who et Virginie Marcucci, en introduction de son étude sur Desperate Housewives, soulignait il y a peu la difficulté à situer l'idéologie partisane d'une série télévisée. ${ }^{11}$ Il n'en demeure pas moins que Doctor Who est essentiellement conçu comme un programme progressiste, peu suspect a priori de prôner un conservatisme presque réactionnaire. Il est ainsi symptomatique qu'en 2012, un épisode-documentaire intitulé « The Science of Doctor Who », interroge des scientifiques britanniques qui évoquent l'enthousiasme que Doctor Who éveille en eux et commentent les technologies présentes dans la série.

Sensible à cette difficulté, Lindy Orthia proposait récemment de la résoudre en distinguant bons et mauvais scientifiques, dans les histoires. La science en elle-même serait essentiellement bonne et ce serait la perversion de tel ou tel scientifique qui conduirait à des résultats catastrophiques. ${ }^{12}$ L'argumentation est fine et précise, mais je ne suis pas certain qu'elle soit entièrement convaincante. En effet, Orthia traite l'ensemble des deux séries, depuis les années 60 jusqu'à nos jours. Or, si la science occupait une place prépondérante dans certaines époques de la première série, la nouvelle fait du Docteur un magicien plutôt qu'un scientifique. ${ }^{13}$ Analyser la seconde série dans la continuité de la première conduit donc à exagérer la place de l'exploration épistémologique. Il me semble que contrairement aux conclusions optimistes de Lindy Orthia, la nouvelle série, à la différence peut-être de la série originelle, envisage la techno-science comme un ensemble imaginaire à l'égard duquel les

${ }^{10}$ MCKeE, A., « Is Doctor Who Political ? », European Journal of Cultural Studies, vol. 7, n ${ }^{\circ} 2,2004$, p. 201-217.

${ }^{11}$ Marcucci, V., Desperate Housewives : un plaisir coupable, Paris, Presses Universitaires de France, 2012.

${ }^{12}$ ORTHIA, L., « Antirationalist critic or fifth column of scientism ? Challenges from Doctor Who to the mad scientist trope ", Public understanding of science, vol. 20, $\mathrm{n}^{\circ} 4,2011, \mathrm{p}$. 525-542.

${ }^{13}$ Rustin, M. et M., « The Regeneration of Doctor Who », J. Plastow (dir.), Children's Literature Annual $n^{\circ}$. The Story and the Self : Some Psychoanalytic Perspectives, Hatfield, University of Hertfordshire Press, 2008, p. 142-159. 
personnages développent des positions éthiques et morales. La techno-science des nouveaux Doctor Who produit ses monstres avec un systématisme qui laisse peu de place à l'ambiguïté.

Est-ce à dire qu'aucune science n'est possible et que la limitation éthique de l'être humain à sa condition de finitude mortelle lui interdit tout développement ? En d'autres termes, la stabilité de l'essence spirituelle et corporelle de l'humanité (un corps biologique mortel qui souffre et qui aime) implique-t-il la stabilité des circonstances de son existence ? Les choses ne sont pas aussi nettes que cela. Si l'ensemble du dispositif signifiant de la série condamne systématiquement les techniques qui permettent de s'affranchir de la mort et de la souffrance, le Docteur chante à de nombreuses reprises les louanges des scientifiques qui explorent l'inconnu. Dans « Waters of Mars » (4.16), il s'extasie sur la soif de découverte des premiers colons de Mars ; dans « The Impossible Planet» (2.8), il admire le courage d'une équipe de scientifiques. Ce que ces équipes d'explorateurs ont en commun, c'est une volonté de découvrir de nouvelles connaissances et le courage de se sacrifier lorsque l'expédition scientifique tourne mal. Il y a donc bien, comme le suggérait Orthia, de bons et de mauvais savants dans les nouveaux Doctor Who, mais la distinction ne passe pas à l'intérieur d'un ensemble de processus unique, la science. Elle sépare deux domaines : d'un côté, la technique, qui cherche à produire des effets sur le monde, de l'autre la science, qui cherche à connaître le monde. C'est la technique, non la science qui est condamnée.

La technique est ainsi présentée comme une volonté de puissance : pour les scientifiques qui développent des inventions aux applications concrètes, le but est de modifier le monde, la matière et les corps. Tout au contraire, la science est un exercice d'abnégation : pour les scientifiques qui mènent des expéditions, le but est d'accroître la somme des connaissances communes. Le profit purement scientifique, on le voit, est d'ordre éthique et il ne participe pas réellement d'une spécificité du scientifique en tant qu'il maîtrise un ensemble de processus épistémiques déterminés, une manière de formuler des hypothèses et des protocoles pour les tester, mais en tant qu'il est un être humain caractérisé par ses émotions : le courage, la curiosité, l'amour de son prochain. De sorte que si le scientifique fou ne constitue pas nécessairement, comme le rappelle Orthia, une condamnation de la science, le scientifique valeureux n'en est pas non plus l'incarnation glorieuse.

\section{Remarques finales}

Du point de vue scientifique, le bilan de la série n'est guère engageant. Si le Docteur est un savant parfait, force est de constater que les processus de son savoir sont entièrement dissimulés au téléspectateur, au point qu'il paraisse plutôt magicien que scientifique. Plus généralement, la nouvelle série témoigne d'une indifférence presque totale au fonctionnement interne des disciplines scientifiques et les envisage bien plutôt comme un ensemble de méthodes et un corpus de connaissances à peu près homogènes. La seule distinction fondamentale est celle qui sépare la techno-science de la science pure.

La techno-science est représentée comme une quête de pouvoir et d'effectivité. Elle est la discipline du scientifique qui cherche à s'augmenter lui-même, afin de dépasser les limites essentielles de la condition humaine. Elle est donc par excellence le territoire du posthumain et constitue, du point de vue naturaliste et statique de l'idéologie à l'œuvre dans la série, une transgression dont la fin ultime est le Mal absolu, incarné par les Daleks. Le bestiaire de la techno-science est celui des machines et des monstres. Là, le corps du 
comédien est effacé par le masque métallique, le maquillage et les effets spéciaux. Son inhumanité est rendue encore plus sensible par la place réservée au jeu des compagnes.

La science pure, elle, est une aspiration noble au savoir pour lui-même, indépendamment de toute considération pratique. On n'aspire qu'à ce que l'on ne possède pas et le revers de la science pure est l'aveu d'une ignorance fondamentale, rarement comblée par la série. La science pure est par conséquent à peu près ineffective. Elle ne change ni la nature humaine, qui est présentée comme immuable, ni même la matérialité du monde. A tout instant, les savants qui la servent sont susceptibles de sacrifier leur propre vie en acceptant la mort et la souffrance comme des traits propres à l'humanité. L'éthique de l'humanité est donc celle d'une puissance à être, jamais actualisée.

Il est difficile pour l'analyste des séries télévisées de ne pas demeurer songeur devant de semblables conclusions. Habitué, à la fois par les nécessités d'une justification académique et par la tradition du champ, à défendre la valeur intellectuelle de son objet d'études, à faire de Buffy l'incarnation d'un merveilleux féminisme et de Doctor Who celle d'un louable humanisme, l'analyste court le risque de commettre, je crois, deux erreurs. La première est de surestimer la subversion qu'un objet médiatique de grande consommation est capable de mettre en œuvre - ce qui ne veut certes pas dire que le discours qui interprète cet objet n'est pas en mesure d'en faire le véhicule de la subversion. La seconde est de faire reposer la valeur d'un système sémiotiquement complexe, qui se sert de discours et d'images, sur son seul contenu, comme si la forme n'existait que pour transmettre un message et qu'un récit audiovisuel n'était jamais qu'un essai politico-moral. C'est pour ces raisons que j'ai essayé de montrer ici, dans la mesure du possible, à la fois que l'interprétation pouvait tirer d'un discours non-subversif matière à réflexion et que ce discours non-subversif pouvait être mis en œuvre avec un grand art, à travers des réseaux complexes et habilement organisés de répliques et d'images, où se rencontraient de multiples techniques artistiques, du jeu du comédien au talent du maquilleur. Que la vertu de la Princesse de Clèves puisse faire frémir qui songe à l'idéologie qui la soutient n'empêche pas que les phrases n'en soient belles.

François-Ronan Dubois Université Stendhal — Grenoble 3

\section{Bibliographie}

CATELlin, S., « Le recours à la science-fiction dans le débat public sur les nanotechnologies : anticipation et prospective », Quaderni, n61, 2006, p. 13-24.

CRANNY-FrANCIS, A., " Mediating Human-technology Relationships : Explorations of Hybridity, Humanity and Embodiment in Doctor Who », Forum, n`8, 2009, en ligne.

Geraghty, L., «From Balaclavas to Jumpsuits : The Multiple Histories and Identities of Doctor Who’s Cybermen », Atlantis, vol. 30, n¹, 2008, p. 85-100.

MARCucCI, V., Desperate Housewives : un plaisir coupable, Paris, Presses Universitaires de France, 2012. 
MCKeE, A., « Is Doctor Who Political ? », European Journal of Cultural Studies, vol. 7, n², 2004, p. 201-217.

ORTHIA, L., "Enlightenment was the choice : Doctor Who and the democratisation of science », Canberra, Thèse de doctorat, 2010.

ORTHIA, L., " Antirationalist critic or fifth column of scientism ? Challenges from Doctor Who to the mad scientist trope ", Public understanding of science, vol. 20, $\mathrm{n}^{\circ} 4,2011, \mathrm{p}$. $525-542$.

QuET, M., « La critique des technologies émergeantes face à la communication promettante. Contestations autour des nanotechnologies », Réseaux, n¹73-174, 2012-2013, p. 271-301.

Rustin, M. et M., " The Regeneration of Doctor Who », J. Plastow (dir.), Children's Literature Annual $n^{\circ}$. The Story and the Self : Some Psychoanalytic Perspectives, Hatfield, University of Hertfordshire Press, 2008, p. 142-159.

Tulloch, J. et Alvarado, M, Doctor Who : The Unfolding Text, Londres, Macmillian Publishers, 1983. 